Enfermagem Brasil 2018;17(4):336-45

\title{
ARTIGO ORIGINAL \\ Fatores de risco/causais para insuficiência renal aguda em adultos internados em terapia intensiva
}

Daniele Lopes*, Letícia da Silva Schran**, João Lucas Campos de Oliveira, D.Sc. ${ }^{* *}$, Rafaela Bramatti Silva Razini Oliveira, M.Sc. ${ }^{* * *}$, Luciana Magnani Fernandes, D.Sc. ${ }^{* * * *}$

*Enfermeira, Pós-Graduanda do Programa de Residência em Gerenciamento de Enfermagem em Clínica Médica e Cirúrgica da Universidade Estadual do Oeste do Paraná (UNIOESTE), Cascavel/PR, ${ }^{* *}$ Enfermeira, Pós-Graduanda do Programa de Residência em Gerenciamento de Enfermagem em Clínica Médica e Cirúrgica da Universidade Estadual do Oeste do Paraná (UNIOESTE), Cascavel/PR, ${ }^{\star \star \star}$ Enfermeiro, Docente colaborador dos cursos de Graduação em Enfermagem e Residência em Gerenciamento de Enfermagem em Clínica Médica e Cirúrgica da Universidade Estadual do Oeste do Paraná (UNIOESTE), Cascavel/PR, ${ }^{* * * \star}$ Enfermeira, Coordenadora do curso de Graduação em Enfermagem da Faculdade Assis Gurgacz (FAG), Cascavel/PR, ${ }^{* * * * *}$ Enfermeira, Professora associada ao Colegiado de Enfermagem da Universidade Estadual do Oeste do Paraná (UNIOESTE), Cascavel/ PR

Recebido em 28 de setembro de 2017; aceito em 21 de janeiro de 2018.

Endereço para correspondência: Letícia da Silva Schran, Rua Aimorés, 562 Santo Onofre 85806330 Cascavel PR, E-mail: le_schran@hotmail.com; Daniele Lopes: lopes.daniele@outlook.com; João Lucas Campos de Oliveira: enfjoaolcampos@yahoo.com.br; Rafaela Bramatti Silva Razini Oliveira: rafaelabramatti@hotmail.com; Luciana Magnani Fernandes: lumagna@terra.com.br

\section{Resumo}

Objetivo: Identificar principais fatores de risco/causais para Insuficiência Renal Aguda (IRA) entre adultos em tratamento hospitalar intensivo e hemodiálise. Material e métodos: Estudo transversal, descritivo, retrospectivo e documental com abordagem quantitativa. Analisaram-se 77 prontuários de pacientes elegíveis que estiveram internados no período de janeiro de 2015 a dezembro de 2016. Aos dados tabulados, procedeu-se análise estatística descritiva. Resultados: A amostra era prevalente do sexo masculino (55,84\%). Quanto aos fatores de risco para IRA, foram identificados, entre outros: hipertensão arterial sistêmica $(36,36 \%)$; diabetes mellitus $(20,78 \%)$ e ventilação mecânica (100\%). Houve uso de droga vasoativa em $61 \%$ da amostra, e apenas nove pacientes não usaram antibióticos. As complicações dialíticas ocorreram em $40,26 \%$ dos casos, com enfoque para hipotensão arterial. A taxa de moralidade foi de $71,43 \%$. Conclusão: Os fatores de risco para IRA são diversos, enfatizados em comorbidades clínicas e itens relacionados ao tratamento intensivo.

Palavras-chave: lesão renal aguda, diálise renal, unidades de terapia intensiva, fatores de risco, cuidados de enfermagem.

\begin{abstract}
Risk/causal factors for acute renal insufficiency in adults in intensive therapy

Objective: To identify the main risk/causal factors for Acute Renal Failure (ARF) among adults in intensive hospital treatment and hemodialysis. Methods: cross-sectional, descriptive, retrospective and documentary study with a quantitative approach. We analyzed 77 charts of eligible patients who were hospitalized in the period from January 2015 to December 2016. To the tabulated data, a descriptive statistical analysis was performed. Results: the sample was prevalent male $(55.84 \%)$. Regarding the risk factors for ARF, we identified, among others: systemic arterial hypertension (36.36\%); diabetes mellitus $(20.78 \%)$ and mechanical ventilation $(100 \%)$. There was vasoactive drug use in $61 \%$ of the sample, and only nine patients did not use antibiotics. Dialytic complications occurred in $40.26 \%$ of the cases, with a focus on hypotension. The morality rate was $71.43 \%$. Conclusion: The risk factors for ARI are diverse, emphasized in clinical comorbidities and items related to intensive care.
\end{abstract}

Key-words: acute kidney injury, renal dialysis, intensive care units, risk factors, nursing care. 


\section{Resumen \\ Factores de riesgo/causales para insuficiencia renal aguda en adultos internados en terapia intensiva}

Objetivo: Identificar principales factores de riesgo/causales para la Insuficiencia Renal Aguda (IRA) entre adultos en tratamiento hospitalario intensivo y hemodiálisis. Material y métodos: Estudio transversal, descriptivo, retrospectivo y documental con abordaje cuantitativo. Se analizaron 77 prontuarios de pacientes elegibles que estuvieron internados en el período de enero de 2015 a diciembre de 2016. A los datos tabulados, se procedió a análisis estadístico descriptivo. Resultados: La muestra era prevalente del sexo masculino $(55,84 \%)$. En cuanto a los factores de riesgo para IRA, fueron identificados, entre otros: hipertensión arterial sistémica $(36,36 \%)$; diabetes mellitus $(20,78 \%)$ y ventilación mecánica $(100 \%)$. Hubo uso de droga vasoactiva en el $61 \%$ de la muestra, y sólo nueve pacientes no usaron antibióticos. Las complicaciones dialíticas ocurrieron en el $40,26 \%$ de los casos, con enfoque para hipotensión arterial. La tasa de moralidad fue de $71,43 \%$. Conclusión: Los factores de riesgo para IRA son diversos, enfatizados en comorbilidades clínicas e ítems relacionados al tratamiento intensivo.

Palabras-clave: lesión renal aguda, diálisis renal, unidades de terapia intensiva, factores de riesgo, cuidados de enfermería.

\section{Introdução}

Alguns tipos de tratamento e, principalmente, hospitalizações por longos períodos podem levar a complicações correlatas à situação clínica do indivíduo, ao exemplo da doença renal (DR), que pode se manifestar aguda ou cronicamente [1,2]. A DR ocorre quando os rins se tornam incapazes de remover produtos de degradação metabólica ou de realizar suas funções reguladoras de volume de líquidos - que culminam na excreção urinária - o que determina a necessidade de terapias de substituição da função renal [1-3].

A Insuficiência Renal Aguda (IRA) é uma das complicações mais comuns no ambiente hospitalar e sua incidência varia de acordo com a gravidade clínica do paciente [4]. É uma patologia complexa, de etiologias múltiplas, caracterizada pela rápida queda da capacidade dos rins em retirar os produtos de degradação do organismo, o que causa distúrbios hídricos, eletrolíticos e acidobásicos [1-4].

Com o avançar da idade, a progressão da lesão vascular pode ser um fator de risco importante para IRA, já que esta pode estar relacionada a outros fatores, como a hipertensão arterial sistêmica (HAS) e aterosclerose, sendo essas doenças mais comuns em idosos [5]. Ademais, a nefrotoxicidade também é considerada um fator de risco para o desenvolvimento da IRA, uma vez que causa alterações renais, funcionais ou estruturais decorrentes das ações de produtos químicos [6].

O tratamento inicial da IRA varia de acordo com o grau de acometimento da doença. As medidas terapêuticas iniciais devem estar voltadas para a correção de volemia, restabelecimento do equilíbrio eletrolítico, controle das manifestações urêmicas e um rigoroso controle hidroeletrolítico e nutricional, além da correção do distúrbio acidobásico [2]. Quando estas condutas terapêuticas se tornam insuficientes ou incapazes de manter uma condição clínica compatível com a vida, a terapia renal substitutiva deve ser implementada através da diálise peritoneal ou da hemodiálise [2,7].

A hemodiálise como terapia de substituição renal é mais amplamente difundida nos serviços clínicos ambulatoriais e hospitalares, incluindo as Unidades de Terapia Intensiva (UTI) no manejo do doente gravemente enfermo e acometido pela injúria renal, contudo, a mortalidade da IRA dialítica é muito superior se comparada à não dialítica [7].

As complicações mais frequentes nas sessões de hemodiálise são: hipo e/ou hipertensão arterial, câimbras, náuseas, vômitos, cefaleia, arritmias cardíacas, prurido, dores lombar e torácica [8]. Essas complicações podem ser casuais e/ou de fácil manejo, o que depende das condições clínicas do paciente. Todavia, podem ser fatais, o que evidencia a necessidade de assistência de enfermagem atenta e precisa aos pacientes durante o período dialítico, visando a integralidade e segurança no cuidado sistematizado [1,9].

A assistência de enfermagem ao doente renal deve basear-se, inclusive, na monitorização das complicações, participar no tratamento das emergências hidroeletrolíticas, avaliar a evolução do paciente ao tratamento e fornecer o apoio físico e emocional, coadunando ao cuidado individualizado [9]. Para que isso aconteça, deve-se ter claro que o processo de enfermagem necessita de muita dedicação [5]. 
Posta a necessidade e a importância de tratar e prevenir a IRA, principalmente no contexto de UTI, postula-se que o conhecimento de fatores de risco que determinam a causalidade da doença nos serviços de cuidados intensivos é muito relevante, pois tende a favorecer a prática baseada em evidências e a viabilização de diretrizes para condutas/cuidados de forma respaldada. Dito isso, os estudos na problemática enunciada se tornam inegavelmente relevantes, culminando-se à pergunta: Quais são os principais fatores de riscos/causais para IRA em adultos internados em UTI que tiveram hemodiálise como tratamento? Portanto, objetivou-se identificar principais fatores de risco/causais para IRA entre adultos em tratamento hospitalar intensivo e hemodiálise.

\section{Material e métodos}

Trata-se de um estudo transversal, descritivo, retrospectivo, documental de abordagem quantitativa. Foi realizado na UTI para adultos de um hospital universitário público do interior do Paraná, Brasil, que conta com 210 leitos exclusivos ao Sistema Único de Saúde (SUS). Por sua vez, a UTI de inquérito possui 14 leitos de tratamento intensivo geral, sendo cinco destinados à hemodiálise, conforme necessidade da clientela.

A população foi composta pelos prontuários dos pacientes internados na UTI no período de janeiro de 2015 a dezembro de 2016. A amostra compreendeu os sujeitos que atenderam os seguintes critérios de elegibilidade: ter o diagnóstico de IRA sem histórico da doença crônica; usado hemodiálise como item de tratamento; e ter 18 anos ou mais de idade. Recrutou-se o total de 102 prontuários, e, aplicando-se os critérios de inclusão/exclusão, 15 pacientes foram excluídos da pesquisa, pois cinco deles tinham menos de 18 anos de idade e 10 já tinham um diagnóstico prévio de Insuficiência Renal Crônica (IRC). Não houve cálculo amostral, pois a intenção foi atingir um senso da população atendida no recorte temporal estabelecido. Logo, a amostra constou de 77 prontuários de pacientes assistidos na UTI no período.

Para definição dos fatores de risco para IRA, primeiramente foi realizado um levantamento bibliográfico de estudos publicados em português sendo encontrados 61 estudos sobre o assunto estudado nos seguintes bancos de dados: Literatura Latino-Americana e do Caribe em Ciências da Saúde (LILACS), Scientific Electronic Library Online (SciELO), Base de Dados de Enfermagem (BDENF), Literatura Internacional em Ciências da Saúde (MEDLINE), Biblioteca Cochrane e Índice Bibliográfico Espanhol de Ciências da Saúde (IBECS).

Para subsidiar o levantamento bibliográfico foram usados os seguintes descritores: Hemodiálise, Unidades Hospitalares de Hemodiálise, Lesão Renal Aguda, Unidade de Terapia Intensiva Adulto, Insuficiência Renal, Fisiologia Renal, Cuidados de Enfermagem, pesquisados nos dicionários de Descritores em Ciência da Saúde (DeCS), juntamente com o conector booleano AND. Foram encontrados 61 estudos publicados sobre o assunto estudado. Este levantamento permitiu a identificação prévia dos fatores de risco mais comuns para a IRA em pacientes internados em UTI. Como o levantamento da literatura não correspondeu ao objetivo fixado deste estudo, optou-se por não descrever os seus resultados detalhadamente, uma vez que este processo foi usado a fim de obter consistência na identificação dos fatores de risco para IRA na pesquisa de campo.

Os dados foram coletados entre maio e junho de 2017. Para isso, foi elaborado um formulário próprio para a extração das seguintes variáveis: sexo; idade; fatores de risco para IRA (incluindo comorbidades e intervenções clínicas); informações acerca da hemodiálise (no de sessões e intercorrências) e desfecho do quadro paciente (alta da UTI; transferência ou óbito).

Após a coleta, os dados foram registrados em planilhas eletrônicas do software Microsoft Office Excel, versão 2010. Neste aparato tecnológico, empregou-se a análise dos dados por meio de estatística descritiva em medidas de proporção. Além disso, para análise das variáveis quantitativas foram calculadas medidas de tendência central e amplitude.

Ressalta-se que este estudo foi submetido e aprovado por Comitê de Ética em Pesquisa institucional, levando-se em consideração as normas éticas para pesquisas envolvendo seres humanos. Logo, recebeu parecer de número 2.072.098/2017 e está registrado nacionalmente por CAAE: 67953717.9.0000.0107. 
Da amostra de 77 prontuários, $55,84 \%$ pacientes $(n=43)$ eram do sexo masculino, e os demais $44,16 \%(n=34)$ do sexo feminino. A média de idade foi de 54,76 anos para as mulheres com variância entre 25 e 80 anos e de 47,67 anos para os homens, com variabilidade entre 18 e 78 anos. A faixa etária de maior prevalência foi de 41 a 50 anos (29,41\%). Do total, $36,36 \%(n=28)$ dos pacientes apresentaram mais de 10 dias de internação hospitalar antes de iniciarem a hemodiálise, sendo o tempo médio de permanência na UTI de 10,5 dias.

Foram identificados três principais diagnósticos médicos na internação dos pacientes, a saber: dor abdominal a esclarecer $(15,58 \% ; n=12)$; rebaixamento de nível de consciência, com proporção de $12 \%(n=9)$ e Trauma Cranioencefálico em $6 \%$ da amostra $(n=5)$. A Tabela 1 descreve os achados referentes aos principais fatores de risco para IRA constatados na amostra dos pacientes.

Tabela I - Distribuição dos fatores de risco para IRA entre pacientes submetidos à hemodiálise, por sexo. Cascavel, 2017.

\begin{tabular}{lllll}
\hline Fatores de risco & \multicolumn{2}{l}{ Feminino } & \multicolumn{2}{l}{ Masculino } \\
\cline { 2 - 5 } Cardiopatias & $\mathbf{n}$ & $\%$ & $\mathbf{n}$ & $\%$ \\
Choque séptico & 3 & 8,82 & 1 & 2,32 \\
Diabetes Mellitus & 1 & 2,94 & 1 & 2,32 \\
Doença Pulmonar Obstrutiva Crônica & 10 & 29,41 & 6 & 13,95 \\
Etilismo & 1 & 2,94 & 3 & 6,98 \\
Hipertensão Arterial Sistêmica & 2 & 5,88 & 6 & 13,95 \\
HIV & 15 & 44,11 & 13 & 30,23 \\
Obesidade & - & - & 1 & 2,32 \\
Politrauma & 2 & 5,88 & 2 & 4,65 \\
Procedimentos cirúrgicos & 3 & 8,82 & 4 & 9,30 \\
Tabagismo & 9 & 26,47 & 5 & 11,62 \\
\hline Ventilação Mecânica & 2 & 5,88 & 9 & 20,93 \\
\hline Fonte: Dados da pesquisa, 2017. & 34 & 100 & 43 & 100 \\
\hline
\end{tabular}

Os níveis de creatinina dos pacientes foram avaliados no primeiro dia de internação hospitalar, e a média da creatinina para os homens no momento da internação foi de $2,4 \mathrm{ml} / \mathrm{dl}$, com variância entre $0,75 \mathrm{ml} / \mathrm{dl}$ e $7,78 \mathrm{ml} / \mathrm{dl}$. Para as mulheres a média foi de 2,26 $\mathrm{ml} / \mathrm{dl}$, com variância entre $0,65 \mathrm{ml} / \mathrm{dl}$ e 15,89 ml/dl. Dados desta ordem são descritos na Tabela II.

Tabela II - Distribuição dos exames laboratoriais entre pacientes com IRA submetidos à hemodiálise, por sexo. Cascavel, 2017.

\begin{tabular}{lllll}
\hline \multirow{2}{*}{ Exames laboratoriais } & \multicolumn{3}{l}{ Sexo } & \multicolumn{2}{l}{ Feminino } \\
\cline { 2 - 5 } & \multicolumn{2}{l}{ Masculino } & $\mathbf{n}$ & $\%$ \\
\cline { 2 - 5 } & $\mathbf{n}$ & $\%$ & 8 & 23,53 \\
\hline Creatinina alterada & 16 & 37,21 & 26 & 76,47 \\
Creatinina normal & 27 & 62,79 & 27 & 79,41 \\
Diurese normal & 33 & 76,75 & 4 & 11,76 \\
Anúria & 6 & 13,95 & 2 & 5,88 \\
Oligúria & 2 & 4,65 & 1 & 2,95 \\
Não consta & 2 & 4,65 & & \\
\hline
\end{tabular}

Fonte: Dados da pesquisa, 2017.

Entre os pacientes estudados, $61 \%(n=77)$ fez uso de pelo menos um tipo de droga vasoativa (DVA), sendo a Noradrenalina $(90,9 \%)$, a droga principal de escolha. Ainda, pôde-se observar que a maior parte dos pacientes $(n=38)$ fez uso de 2 ou 3 tipos de antibióticos, o que está ilustrado na Tabela III.

Tabela III - Distribuição dos pacientes com IRA submetidos à hemodiálise, de acordo com número de antibióticos recebidos. Cascavel, 2017. 


\begin{tabular}{lll}
\hline № de antibióticos & $\mathbf{n}$ & $\%$ \\
\hline 0 & 9 & 11,69 \\
1 & 17 & 22,08 \\
2 & 20 & 25,97 \\
3 & 18 & 23,38 \\
4 & 6 & 7,79 \\
5 & 4 & 5,19 \\
Mais de 5 & 3 & 3,90 \\
Total & 77 & 100 \\
\hline
\end{tabular}

Fonte: Dados da pesquisa, 2017.

Por sua vez, a Tabela IV descreve quais antibióticos foram empregados, em sua frequência, no tratamento dos pacientes com IRA. O mesmo paciente pode ter usado diversos medicamentos.

Tabela IV - Distribuição dos antibióticos empregados no tratamento dos pacientes com IRA submetidos à hemodiálise, por sexo. Cascavel, 2017.

\begin{tabular}{lllll}
\hline \multirow{2}{*}{ Antibióticos } & \multicolumn{2}{l}{ Feminino } & \multicolumn{2}{l}{ Masculino } \\
\cline { 2 - 5 } & $\mathbf{n}$ & $\%$ & $\mathbf{n}$ & $\%$ \\
\hline Não Usou & 3 & 8,82 & 6 & 13,95 \\
Aciclovir & 1 & 2,94 & - & - \\
Amicacina & 2 & 5,88 & 1 & 2,32 \\
Amoxacilina & 1 & 2,94 & - & - \\
Azitromicina & 6 & 17,64 & 8 & 18,60 \\
Carbamazepina & - & - & 1 & 2,32 \\
Cefazolina & 1 & 2,94 & 3 & 6,98 \\
Cefepime & 16 & 47,05 & 16 & 37,21 \\
Ceftriaxona & 11 & 32,35 & 16 & 37,21 \\
Ceftazidima & 2 & 5,88 & - & - \\
Ciprofloxacino & 6 & 17,64 & 5 & 11,63 \\
Clindamicina & 3 & 8,82 & 3 & 6,98 \\
Eritromicina & 2 & 5,88 & 3 & 6,98 \\
Gentamicina & - & - & 1 & 2,32 \\
Linezolida & 2 & 5,88 & 3 & 6,98 \\
Meropenem & 11 & 32,35 & 10 & 23,25 \\
Moxifloxacino & - & - & 1 & 2,32 \\
Piperacilina & 2 & 5,88 & 1 & 2,32 \\
Pipetazo & 3 & 8,82 & 1 & 2,32 \\
Polimixina B & 3 & 8,82 & 4 & 9,30 \\
Tiamina & 2 & 5,88 & 1 & 2,32 \\
Teicoplanina & 7 & 20,59 & 3 & 6,98 \\
Tigeciclina & - & - & 3 & 6,98 \\
Vancomicina & - & - & 4 & 9,30 \\
\hline
\end{tabular}

Fonte: Dados da pesquisa, 2017.

Todos os pacientes (100\%) realizaram sessões de hemodiálise. Observa-se que a maior prevalência 25,97\% $(n=20)$ dos pacientes realizou mais de 10 sessões durante 0 internamento, seguido por $14,28 \%(n=11)$ com apenas uma sessão e $11,69 \%(n=9)$ com três sessões conforme demonstrado na Tabela V. Vale ressaltar que $100 \%$ dos pacientes que realizaram apenas uma sessão de hemodiálise evoluíram para óbito. 
Tabela V - Distribuição dos pacientes com IRA pela quantidade de sessões de hemodiálise. Cascavel, 2017.

\begin{tabular}{lll}
\hline No de sessões de hemodiálise & $\mathbf{N}$ & $\%$ \\
\hline 1 & 11 & 14,28 \\
2 & 14 & 18,18 \\
3 & 9 & 11,69 \\
4 & 7 & 9,09 \\
5 & 3 & 3,90 \\
6 & 6 & 7,79 \\
7 & 1 & 1,30 \\
8 & 1 & 1,30 \\
9 & 2 & 2,60 \\
10 & 3 & 3,90 \\
Mais de 10 & 20 & 25,97 \\
Total & 77 & 100 \\
\hline Fonte: Dados da pesquisa, 2017 & &
\end{tabular}

No presente estudo, pode-se observar que $40,26 \%(n=31)$ dos pacientes apresentou pelo menos um tipo de complicação durante a sessão de hemodiálise, sendo a hipotensão arterial a de maior prevalência conforme aponta a Tabela VI. Um paciente pode ter apresentado mais de uma intercorrência.

Tabela VI - Distribuição das intercorrências dialíticas entre pacientes com IRA submetidos à hemodiálise. Cascavel, 2017.

\begin{tabular}{lll}
\hline Intercorrências & N & $\%$ \\
\hline Arritmia & 4 & 5,19 \\
Bradicardia & 5 & 6,49 \\
Êmese & 1 & 1,30 \\
Esforço respiratório & 10 & 12,99 \\
Hipertensão arterial & 5 & 6,49 \\
Hiperglicemia & 5 & 6,49 \\
Hipertermia & 6 & 7,79 \\
Hipoglicemia & 21 & 27,27 \\
Hipossaturação & 11 & 14,28 \\
Hipotensão & 65 & 84,41 \\
Palidez & 1 & 1,30 \\
Parada cardiorrespiratória & 1 & 1,30 \\
Taquicardia & 15 & 19,48 \\
Taquipneia & 2 & 2,60 \\
Taquirritimia & 1 & 1,30 \\
Tremores & 1 & 1,30
\end{tabular}

Fonte: Dados da pesquisa, 2017.

Quanto ao desfecho do quadro da amostra de pacientes, obteve-se os seguintes achados: um paciente em transferência hospitalar (1,3\%); 21 receberam alta da UTI $(27,27 \%)$; e 55 casos de óbito, obtendo-se mortalidade em $71,43 \%$ dos pacientes com IRA tradados com hemodiálise.

\section{Discussão}

Conforme os dados demonstrados, os homens são muito afetados por este tipo de problema de saúde, a IRA. A despeito da maior vulnerabilidade e das altas taxas de morbimortalidade, os homens não buscam, como as mulheres, os serviços de atenção básica com frequência e periodicidade [10-11]. Este achado pode refletir a maior internação na UTI, e, por consequência, o desenvolvimento de IRA na população masculina. 
O tempo de internação hospitalar depende de vários fatores como, por exemplo, a natureza da doença básica e as exigências terapêuticas decorrentes das complicações, além da evolução clínica, complicações e agravos ao qual o paciente está sujeito. É habitual que o tempo de internação em UTI seja curto, sendo citadas na literatura permanências médias com duração de 1 dia e de 2 a 4 dias; contudo, há internações de 5 a 10 dias [12,13]. Logo, inferese que a média de internação na UTI dos pacientes com IRA foi alta, possivelmente por sua gravidade clínica, inclusive, refletida na elevada mortalidade.

A hipoperfusão renal prolongada é a causa mais frequente de IRA, principalmente entre aqueles que foram submetidos a grandes cirurgias, traumas, hipovolemia grave, sepse e grandes queimados [14]. Ademais, os principais fatores para IRA demonstrados corroboram achados anteriores os quais ratificam que os fatores de risco/causais presentes em pacientes que desenvolveram IRA eram HAS (54,5\%), seguido de DM (27\%) [5].

A ventilação mecânica (VM) constatada em toda a amostra pode gerar consequências imediatas sobre a função renal, como: diminuição de 20 a $40 \%$ do fluxo urinário, balanço hídrico positivo, retenção de sódio, provocando uma queda do débito cardíaco e nas alterações humorais e afetando de forma direta ou indireta a função renal [15].

A VM pode levar à falência renal aguda por três mecanismos, a saber, efeitos nos gases arteriais; efeito sobre o fluxo sanguíneo sistêmico e renal e liberação sistêmica de agentes inflamatórios. A influência sobre a função renal está relacionada com hipoperfusão renal induzida por ventilação mecânica [15]. Destarte, a VM é um importante preditor de mortalidade em pacientes com IRA na UTI, pois ela é uma importante fonte de aparecimento de comorbidades [7-16].

Os dados demonstrados na Tabela II são de grande importância, pois o critério de diagnóstico Risk Injury Failure Loss and End-Stage Renal Failure (RIFLE) se baseia na definição de três estágios de IRA (Risk, Injury e Failure), que têm como base alterações da creatinina sérica juntamente com os valores do débito urinário, configurando os estágios de disfunção renal [17].

A creatinina sérica é um importante marcador do estado nutricional e apresenta relações com a mortalidade de pacientes com enfermidades renais em terapia intensiva [18], bem como o uso de DVA, que apresenta relação significante com o desenvolvimento de IRA pela hipoperfusão e isquemia renal, além de importante fator de risco para mortalidade em terapia intensiva devido à instabilidade hemodinâmica dos pacientes e em função do mecanismo de vasoconstrição [18-19].

A noradrenalina é uma DVA comum em UTI, sendo um vasopressor utilizado em pacientes hipotensos, desde que não hipovolêmicos [13]. Acerca disso, é visível que a amostra esteve exposta aos eventos do vasopressor, o que, conforme notado anteriormente, possui relação direta na perfusão renal, e, consequentemente, predispõe os pacientes à IRA [18-19].

O número de antibióticos utilizados por cada paciente é alarmante, uma vez que os antibióticos podem levar a danos renais pela nefrotoxicidade. Para esta análise, alguns fatores relacionados aos pacientes devem ser levados em consideração como idade, função renal, função hepática, desidratação, dose da droga, associação com outros fármacos [20-22]. No entanto, o uso de antibióticos é uma intervenção rotineira e necessária ao tratamento dos pacientes, sendo imprescindível o seu emprego clínico racional.

A hemodiálise em UTI é indicada para indivíduos que apresentam estados graves de descompensação hidroeletrolítica e edema pulmonar, além de estágios avançados de uremia que, se não tratados imediatamente, podem levar à morte [23]. Várias complicações podem ocorrer durante as sessões de hemodiálise; e, quanto mais sessões, pior o prognóstico do paciente devido à gravidade do paciente, instabilidade hemodinâmica, tipo de IRA e as complicações que podem ocorrer durante as sessões [2]. Isso posto, percebe-se que a amostra realmente apresentava gravidade no quadro de IRA, pois o número de sessões necessárias à hemodiálise foi alto.

Durante as sessões de hemodiálise podem ocorrer algumas complicações que podem ser extremamente graves e até fatais [24]. A hipotensão arterial foi a complicação de maior prevalência durante as sessões de hemodiálise, e trata-se de um reflexo primário da grande quantidade de líquidos que é removida do volume plasmático durante uma sessão rotineira de diálise. Em geral, as causas comuns da hipotensão durante a hemodiálise são: flutuações na velocidade de ultrafiltração; velocidade de ultrafiltração alta; peso seco almejado muito baixo; medicamentos anti-hipertensivos; superaquecimento da solução de diálise; ingestão de alimentos; neuropatia autônoma; isquemia tecidual; disfunção diastólica; frequência cardíaca e 
contratilidade [25]. Com isso, postula-se que conhecer a relação/associação das intercorrências e o perfil dos pacientes pode ser um vislumbre de estudos futuros.

O prognóstico de IRA refere-se predominantemente à mortalidade e, com menor índice, à recuperação da função renal durante a internação hospitalar. A sobrevida é um bom prognóstico de um paciente com IRA e é muito variável, dependendo de fatores que não estejam relacionados diretamente à doença renal [7,23]. Logo, esta assertiva denota claramente o prognóstico clínico ruim dos pacientes assistidos na UTI de inquérito, pois, inegavelmente, a taxa de mortalidade foi alta.

A atuação do enfermeiro e da equipe multidisciplinar visa evitar a IRA como complicação da internação ou minimizar seus efeitos e promover um melhor prognóstico. Algumas estratégias e ações básicas podem ser utilizadas, como, por exemplo: monitorização rigorosa do balanço hidroeletrolítico e precauções quanto a dosagem, administração e interação medicamentosa, principalmente em drogas potencialmente nefrotóxicas [21,22]. Estas ações devem redundar a avaliação diária da função renal do paciente crítico, sendo isso uma ferramenta indispensável ao trabalho qualificado em UTI [26].

O enfermeiro que atua em UTI presta assistência a pacientes com as mais variadas patologias. Por isto, faz-se necessário uma constante renovação e apreensão de diversos conhecimentos para capacitar e qualificar sua equipe. Sobre a IRA, os enfermeiros, por meio do trabalho sistematizado [27] devem, entre outros, se atentar ao quadro clínico dos pacientes e os achados de injúria renal (densidade, volume, índice urinário, ureia, creatinina plasmática e ácido úrico), além de analisar odor, transparência, resíduos e coloração da urina, contribuindo na determinação da etiologia e na prevenção das complicações [2]. Isso é importante porque a deficiência de conhecimento sobre a IRA entre enfermeiros já foi constatada recentemente [28] reforçando que o profissional precisa se munir do saber científico para intervir de maneira mais eficaz às necessidades dos pacientes que sofre da injúria.

A IRA pode trazer como manifestações clínicas, mal-estar inespecífico até um quadro urêmico (náusea, vômitos, sangramento gastrintestinal, tamponamento pericárdico, dispneia, hipertensão, alterações neurológicas e do nível de consciência). Os pacientes podem ter sinais e sintomas decorrentes da doença de base (exemplo: pneumonia com choque séptico, insuficiência cardíaca grave, etc.) ou da perda de função renal. Os sintomas dependem da gravidade da insuficiência renal, de sua velocidade de instalação, bem como de sua etiologia. Como tratam-se de sinais e sintomas inespecíficos e que podem ser facilmente confundidos com outras patologias, faz-se necessário uma anamnese e exame físico completo por parte do enfermeiro e da equipe multidisciplinar, atentando-se principalmente às comorbidades e patologias já existentes, balanço hídrico e análise minuciosa dos exames laboratoriais [7].

O diagnóstico da IRA se dá principalmente pelo método laboratorial no qual é avaliado no sangue a elevação nos níveis de ureia, creatinina, ácido úrico, a acidose metabólica, hipo ou hipernatremia, hiperpotassemia, hipo ou hipercalemia e hiperfosfatemia e anemia normocítica. Na urina, avalia-se a osmolalidade, sódio, creatinina, ureia e sendimentos urinários [23]. Além do diagnóstico médico, reforça-se, com base no conhecimento levantado neste estudo, que a identificação de fatores de risco e aspectos que entornam o tratamento do paciente com IRA é fundamental para que o enfermeiro alavanque estratégias racionais de assistência, se munindo das informações inerentes à doença, mas também, aos recursos necessários para o cuidado integral e individualizado.

Conclusão

Por meio deste estudo foi possível descrever o cenário que envolve o risco/causalidade de IRA em adultos assistidos em regime hospitalar intensivo. Sobressaíram os seguintes achados: maior proporção de homens; alta média de dias de internação na UTI; principais fatores de risco para IRA: hipertensão arterial sistêmica; diabetes mellitus; ventilação mecânica; uso de drogas vasoativas e antibióticos. Face ao tratamento de hemodiálise, os pacientes expõem-se principalmente à hipotensão como intercorrência dialítica. Ademais, a mortalidade constatada foi elevada.

Com base nos achados, conclui-se que os fatores de risco para IRA são diversos, enfatizados em comorbidades clínicas e itens relacionados ao tratamento intensivo. Apesar das limitações do estudo expressas pela impossibilidade de generalizações e ausência de análise estatística inferencial, acredita-se que este estudo dá robustez ao arsenal de conhecimento produzido, e reforça a necessidade de implantação de diretrizes clínicas de cuidado muito bem mapeadas, o que deve incluir a participação do enfermeiro. 
1. Santos ES, Marinho, CMS. Principais causas de insuficiência renal aguda em unidades de terapia intensiva: intervenção de enfermagem. Rev Enf Ref 2013;3(9):181-9.

2. Leite EMD, Araújo ERA, Ira ALB, Silva FS, Oliveira ACF, Lima CF. Perfil clínico de pacientes submetidos à hemodiálise. Monográficos de Investigación en Salud 2013;19:1-7.

3. Guyton AC, Hall JE. Tratado de fisiologia médica. 13로 ed. Rio de Janeiro: Elsevier; 2017.

4. Santos JCO, Mendonça MAO. Fatores predisponentes para lesão renal aguda em pacientes em estado crítico: revisão integrativa. Rev Soc Bras Clin Med 2015;13(1):6974.

5. Triquez SL, Dallacosta FM. Perfil dos pacientes com insuficiência renal aguda na unidade de terapia intensiva e principais diagnósticos de enfermagem. Unoesc \& Ciência - ACBS 2012;3(2):123-130.

6. Manso MEG, Biffi ECA, Gerardi TJ. Prescrição inadequada de medicamentos a idosos portadores de doenças crônicas em um plano de saúde no município de São Paulo, Brasil. Rev Bras Geriatr Gerontol 2015;18(1):151-64.

7. Peres $L A B$, Wandeur $V$, Matsuo $T$. Preditores de injúria renal aguda e de mortalidade em uma Unidade de Terapia Intensiva. J Bras Nefrol 2015;37(1):38-46.

8. Debone MC, Pedruncci ESN, Candido MCP, Marques S, Kusumota L. Diagnósticos de enfermagem em idosos com doença renal crônica em hemodiálise. Rev Bras Enferm. 2017;70(4):833-9.

9. Brunner BS, Suddarth DS. Tratado de enfermagem médico-cirúrgica. 13a ed. Rio de Janeiro: Guanabara Koogan; 2016.

10. Cavalcanti JRD, Ferreira JA, Henriques AHB, Morais GSN, Trigueiro JVS, Torquato IMB. Assistência integral a saúde do homem: necessidades, obstáculos e estratégias de enfrentamento. Esc Anna Nery 2014;18(4):628-34.

11. Pinheiro RS, Viacava F, Travassos C, Brito AS. Gênero, morbidade, acesso e utilização de serviços de saúde no Brasil. Ciênc Saúde Coletiva 2002;7(4):687-707.

12. Prece A, Cervantes J, Mazur CS, Visentin A. Perfil de pacientes em terapia intensiva: necessidade do conhecimento para organização do cuidado. Cad. da Esc. de Saúde. 2016; 2(16):35-48.

13. Melo WF, Pereira AWR, Alves VQ, Saldanha HGAC, Sousa JS. Assistência de enfermagem na urgência e emergência ao paciente vítima de insuficiência renal aguda: uma revisão bibliográfica. REBES 2015;5(2):6-11.

14. Miyahira AJM. Insuficiência renal aguda. Rev Med Hered 2003;14(1):36-47.

15. Santos LL, Magro MCS. Ventilação mecânica e a lesão renal aguda em pacientes na unidade de terapia intensiva. Acta Paul Enferm 2015;28(2):146-51.

16. Santos JCO, Mendonça MAO. Fatores predisponentes para lesão renal aguda em pacientes em estado crítico: revisão integrativa. Rev Soc Bras Clin Med 2015;13(1):6974.

17. Levi TM, Souza SP, Magalhães JG, Carvalho MS, Cunha ALB, Dantas JGAO, et al. Comparação dos critérios RIFLE, AKIN e KDIGO quanto à capacidade de predição de mortalidade em pacientes graves. Rev Bras Ter Intensiva 2013;25(4):290-6.

18. Luft J, Boes AA, Lazzari DD, Nascimento ERP, Busana JA, Canever BP. Lesão renal aguda em unidade de terapia intensiva: características clínicas e desfecho. Cogitare Enferm 2016;21(2):1-9.

19. Guedes JR, Silva ES, Carvalho IN, Oliveira MD. Incidência e fatores predisponentes de insuficiência renal aguda em unidade de terapia intensiva. Cogitare Enferm 2017;22(2):1-9.

20. Pinto PS, Carminatti M, Lacet T, Rodrigues DF, Nogueira LO, Bastos MG, et al. Insuficiência renal aguda nefrotóxica: prevalência, evolução clínica e desfecho. J Bras Nefrol 2009;31(3):183-9.

21. Perazella MA. Renal vunerability to drug toxicity. Clin J Am Soc Nephrol 2009;4(7):1275-83.

22. Duarte F, Pessoa EA, Reis LA, Schor N, Borges FT. Priming previne a insuficiência renal aguda nefrotóxica através da estimulação do mecanismo de defesa antioxidante. J Bras Nefrol 2016;38(2):161-172. 
23. Barbosa KRA, Silva SC, Pope S, Fornari JV, Rodrigues FSM, Banabé AS, et al. Indicações de hemodiálise de emergência em uma unidade de terapia intensiva de um hospital particular de Atibaia -SP. Science in Health 2012;3(3):131-8.

24. Cardoso BG, Carneiro TA, Magro MCS. Recuperação de pacientes com lesão renal aguda dialítica e não dialítica. Cogitare Enferm 2017;22(1):1-9.

25. Castro JM, Ferreira JS, Alves JMC, Herckert MMS, Barbosa R, Cabral FS et al. Intervenções de enfermagem em pacientes com insuficiência renal crônica em tratamentos hemodialítico. BJSCR 2017;19(3):65-67.

26. Cerqueira DP, Tavares JR, Machado RC. Fatores preditivos da insuficiência renal e algoritmo de controle e tratamento. Rev Latinoam Enferm 2014;22(2):211-7.

27. Silva CMS, Silva DAN, Silva GGP, Maia LFS, Oliveira TS. Insuficiência renal aguda: principais causas e a intervenção de enfermagem em UTI. Revista Recien 2016;6(16):48-56.

28. Nascimento RAM, Assunção MSC, Silva Junior JM, Amendola CP, Carvalho TM, Lima $E Q$ et al. Conhecimento do enfermeiro para identificação precoce da injúria renal aguda. Rev Esc Enferm USP 2016;50(3):399-404. 